\title{
Exploration of microplastics from personal care and cosmetic products and its estimated emissions to marine environment: an evidence from Malaysia
}

\begin{abstract}
This study aims understand microplastics from personal care and cosmetic products in Malaysia via quantification and characterization of microplastics together with emission estimation to marine environment. A total of 214 respondents from all over Malaysia were surveyed with identification of top ten personal care and cosmetic products usage. Particles found in facial cleaner/scrub and toothpaste were colored and colorless with majority of granular shapes. Particles in toothpaste were found between 3 and $145 \mu \mathrm{m}$ while particles in facial cleaner/scrub were found to be between 10 and $178 \mu \mathrm{m}$, stipulating the presence of microplastics. Plastic polymers (LDPE and polypropylene) were found in all facial cleaner/scrub samples while only plastic polymers (LDPE) were present in toothpaste sample G. A total of 0.199 trillion microplastics are expected to be released annually to marine environment in Malaysia. Personal care and cosmetic products are seen as one of the microplastics sources for Malaysia and worldwide.
\end{abstract}

Keyword: Personal care and cosmetic products; Microplastics; Microbeads; Emission; Marine environment 